\title{
Mehr als nur Rohstofflieferant
}

\author{
Mit der Transformation zu einer biobasierten \\ Wirtschaft bieten sich auch über die Rohstoff- \\ bereitstellung hinaus Entwicklungsmöglichkeiten \\ für den ländlichen Raum. Diese in einer nach- \\ haltigen Art und Weise zu fördern, ist eine \\ Zukunftsaufgabe von Politik, Wirtschaft, Wissen- \\ schaft und Zivilgesellschaft. \\ Von Johannes Rupp, Katharina Heinbach, \\ Jörg Böhmer und Frank Wagener
}

$\mathrm{T}$ echnische und gesellschaftliche Umwälzungen prägen den ländlichen Raum bis heute und werden ihn auch zukünftig beeinflussen. Der Strukturwandel in ländlichen Räumen ist gekennzeichnet durch den Rückgang an Beschäftigung, die fortschreitende Mechanisierung und Automatisierung von betrieblichen Abläufen sowie die Digitalisierung. Eine Folge davon ist ein zunehmender Preisdruck auf die bereitgestellten Güter und eine fortschreitende Konzentration auf weniger Betriebe. Die Gemeinsame Agrarpolitik (GAP) der Europäischen Union tritt dieser Tatsache nur bedingt entgegen. Landwirtschaftliche Innovationen, wie beispielsweise das „Precision-Farming“, das heißt, zum Beispiel die punktgenaue Versorgung von Äckern mit Nährstoffen, bedürfen größerer Investitionen, die durch kleinere und mittlere Betriebe nicht oder nur schwer zu leisten sind. Gleiches gilt für softwarebasierte Produkte in der Forstwirtschaft, die eine bedarfsgerechte Bereitstellung von Rohholz für die holzverarbeitende Industrie ermöglichen.

Mit der Transformation von einer fossil $\mathrm{zu}$ einer biobasierten Wirtschaft stellt sich die Frage, inwiefern bestehende Wirtschaftsstrukturen im ländlichen Raum von dieser Entwicklung profitieren können. Die Erweiterung der Produktpalette und die Bereitstellung von höherwertigen Produkten bieten potenziell zusätzliche Wertschöpfung und Beschäftigung. Die Förderung der Bioenergie im Strom-, Wärme- und Kraftstoffbereich hat in der ersten Dekade der 2000er Jahre für einen Schub im ländlichen Raum gesorgt. Durch die energetische Biomassenutzung erzielen mittlerweile eine Vielzahl von Akteure/innen in der Land- und Forstwirtschaft einen wirtschaftlichen Nutzen in Form von Einkommen und Gewinnen, vor allem durch den Betrieb von Biogasanlagen, Heizwerken und Nahwärmenetzen. Zudem verbleiben Erträge aus der Beteiligung privater Haushalte oder Einsparungen bei den Heizkosten ebenso im ländlichen Raum wie Zinseinnahmen ortsan- sässiger Banken und geleistete Steuereinnahmen. Unterstützt wurde diese Entwicklung auch durch das Bundesministerium für Ernährung und Landwirtschaft (BMEL) über die Fördermaßnahme Bioenergie-Regionen. Die mehrmalige Novelle des Erneuerbare-Energien-Gesetzes (EEG) führte allerdings in den letzten Jahren zu einem massiven Rückgang an Investitionen in diesem Bereich (Rupp et al. 2017). Von Interesse ist daher, mit welchen Maßnahmen der Fortbestand, der aktuell in Betrieb befindlichen Anlagen gesichert oder sogar eine Weiterentwicklung erzielt werden kann. Interessant ist diese Frage vor dem Hintergrund der Möglichkeiten einer gekoppelten stofflich-energetischen Biomassenutzung. Um an dieser Stelle weiter zu kommen, lohnt sich die Betrachtung der Vielfalt an stofflichen Verwertungspfaden und Anwendungsbereichen, die für den ländlichen Raum von Bedeutung sein könnten.

\section{Möglichkeiten einer ländlichen Bioökonomie}

Eine solche Betrachtung erfolgt im Rahmen des durch das BMEL geförderten Verbundvorhabens „Potenzialfelder einer ländlichen Bioökonomie“. Zu Beginn des Vorhabens wurde dazu die Begriffsbestimmung einer ländlichen Bioökonomie geschärft: Unter der ländlichen Bioökonomie ist die Weiterentwicklung einer Bioökonomie zu verstehen, bei welcher der ländliche Raum nicht nur als Rohstofflieferant für industrielle Bioökonomie-Konzepte betrachtet wird, sondern verstärkt selbst die Umsetzung von dezentralen Bioökonomie-Ansätzen vorantreibt. Dies bedeutet, dass nach Möglichkeit ein Großteil der Wertschöpfungsstufen und -schritte innerhalb von einzelnen Regionen realisiert wird. Damit soll erreicht werden, dass auch der ländliche Raum von den möglichen positiven Effekten einer wachsenden Bioökonomie mit Blick auf Wertschöpfung und Beschäftigung profitiert. Um eine solche Bioökonomie zukünftig zu fördern, ist es notwendig, potenzielle Handlungsspielräume abzustecken. Dazu bedarf es der Betrachtung von Ausgangsstoffen, Märkten sowie der übergeordneten politischen Rahmenbedingungen.

Bei der Betrachtung von Ausgangsrohstoffen für einzelne Verwertungspfade und Anwendungsbereiche einer ländlichen Bioökonomie sind sowohl das aktuell als auch zukünftig verfügbare Rohstoff- und Flächenpotenzial relevant. Hierbei sind mögliche Konkurrenzen und Synergien im Anbau und in der Verwertung der Rohstoffe zu beachten. Wesentliche Basisrohstoffe für die Bioökonomie werden schwerpunktmäßig in der Land- und Forstwirtschaft erzeugt (halmgutartige und holzige Erntegüter). Dazu kommen Sekundärrohstoffe (Rest- und Ab- 
fallstoffe), wie zum Beispiel Gärreste aus der Biogasproduktion, Nebenprodukte aus der Sägeindustrie oder Landschaftspflegematerial. Durch die Vielfalt der Kulturlandschaften in Deutschland stellen sich die jeweiligen Potenziale unterschiedlich dar. So bilden zum Beispiel Grünlandaufwüchse und Holz in den Mittelgebirgslagen einen Schwerpunkt, während verschiedene Acker- und Sonderkulturen, ebenso wie Sekundärrohstoffe aus Biogasanlagen in den landwirtschaftlichen Gunstregionen oder Übergangslagen im Fokus sind. Zudem sorgt der Strukturwandel, belegt durch Betriebsaufgaben oder ungünstig gelegene Grünlandflächen, für weitere frei werdende Potenziale.

Dem Angebot an Rohstoffen steht die Nachfrage bestehender oder sich neu entwickelnder Märkte im Nahrungs- und Futtermittelsektor, in der Energiebereitstellung sowie in der stofflichen Nutzung gegenüber. Bedeutend für eine ländliche Bioökonomie sind hier Verwertungspfade und Produkte, für die es nicht nur eine heimische Rohstoffbasis, sondern auch eine konkurrenzfähige Erst- und idealerweise Weiterverarbeitung der Rohstoffe im ländlichen Raum gibt. Ein interessanter Markt ist die Aufbereitung von Gärresten zu Düngemitteln. Diese können vor Ort hergestellt und in der Landwirtschaft oder im Gartenbau auch regional abgesetzt werden. Ein weiterer interessanter Absatzmarkt ist die Herstellung von Verbundwerkstoffen unter Einsatz von Naturfasern, in etwa Hanfund Holzfasern. Hier sind vor allem Regionen interessant, die bereits über eine Kunststoffverarbeitung verfügen und in der Nähe potenzieller Abnehmer/innen wie beispielsweise der Automobilindustrie liegen. Weitere Absatzmöglichkeiten liegen verstärkt auch in der Elektro- und Möbelindustrie. Bezogen auf den Auf- und Ausbau von dezentralen Erstverarbeitungsstrukturen befinden sich zusätzlich interessante Märkte in der Verarbeitung von biobasierten Fasern zu Dämmstoffen und Holzersatzwerkstoffen im Bereich Bauen und Wohnen sowie zu Produkten der Papierindustrie, beispielsweise Papier, Pappe oder Kartonagen. Bestehende Hemmnisse für die Entwicklung dieser Märkte sind der teilweise deutlich höhere Preis gegenüber mineralischen und fossilen Referenzprodukten, wie im Fall der biobasierten Dämmstoffe, oder noch unzureichende Markterfahrungen, wie im Fall der Herstellung von Produkten der Papierindustrie aus Grasfasern. Hier bedarf es der weiteren Ansprache von auf Umweltbelange orientierten Abnehmer/innen sowie der Kommunikation und Kooperation mit etablierten Marktakteur/innen. Die Herstellung von biobasierten Chemikalien sowie Biokunststoffen ist für eine ländliche Bioökonomie derzeit weniger interessant. Große (petrochemische) Konzerne dominieren diesen Markt. Zudem erfordert die Herstellung dieser Produkte technisch anspruchsvolle Verfahren, eine entsprechende Verkehrsinfrastruktur sowie umfassende Investitionen. Dadurch sind viele Prozesse dezentral im ländlichen Raum kaum realisierbar.

Die zukünftige Entwicklung einer ländlichen Bioökonomie bedarf der zusätzlichen politischen Steuerung, um eine Verbesserung der ökonomischen und ökologischen Nachhaltigkeit der Urproduktion und des verarbeitenden Gewerbes - wie in den übergeordneten Zielen der europäischen Bioökonomiestrategie formuliert (Europäische Kommission 2012) - zu erreichen. Auf nationaler Ebene gibt es noch keinen explizit formulierten politischen Rahmen für eine ländliche Bioökonomie. Lediglich über die Abdeckung verschiedener Politikbereiche, wie zum Beispiel der Agrar-, Energie-, Umwelt-, Klimasowie Wirtschaftspolitik, können Schlussfolgerungen für eine ländliche Bioökonomie gezogen werden. Die hier getroffenen Entscheidungen haben Auswirkungen auf das Handeln der Akteur/innen und die Rahmenbedingungen im ländlichen Raum und bedürfen zukünftig einer größeren Aufmerksamkeit.

Um eine ländliche Bioökonomie im Sinne der Nachhaltigkeitsziele über eine gezielte politische Steuerung zu fördern, bedarf es geeigneter Handlungsspielräume auf regionaler Ebene und mehr guter Beispiele. Zugleich müssen die ökologischen und ökonomischen Folgen und Chancen einer solchen Entwicklung für Akteur/innen in Verwaltung und Politik, Wirtschaft und Zivilgesellschaft transparent gemacht werden. Dies kann durch eine exemplarische Veranschaulichung von Beschäftigungs- und Wertschöpfungspotenzialen sowie ökobilanziellen Betrachtungen erfolgen. Darüber hinaus ist die Vernetzung von Innovationsträger/innen und die Begleitung von Pionieraktivitäten sinnvoll. Diese dienen der besseren Sichtbarkeit und Verbreitung bestehender Ansätze in verschiedenen Regionen und Wirtschaftsbereichen.

\section{Literatur}

Europäische Kommission (2012): Innovating for sustainable growth: a bioeconomy for Europe. Brüssel.

Rupp, J. et al. (2017): Ermittlung der Wertschöpfungs- und Beschäftigungseffekte in drei ausgewählten Bioenergie-Regionen. Schriftenreihe des IÖW 214/17. Berlin.

\section{AUTOR/INNEN + KONTAKT}

Johannes Rupp und Katharina Heinbach sind wissenschaftlicher Mitarbeiter /innen im Forschungsfeld Nachhaltige Energiewirtschaft und Klimaschutz am IÖW.

Institut für ökologische Wirtschaftsforschung, Potsdamer Str. 105, 10785 Berlin. E-Mail: johannes.rupp@ioew.de, katharina.heinbach@ioew.de

Jörg Böhmer ist stellvertretender Leiter und Frank Wagener Leiter des Arbeitsbereichs Biomasse und Kulturlandschaftsentwicklung am Institut für angewandtes Stoffstrommanagement (IfaS).

IfaS, Hochschule Trier, Umwelt-Campus Birkenfeld, Postfach 1380, 55761 Birkenfeld. E-Mail: j.boehmer@umwelt-campus.de, f.wagener@umwelt-campus.de
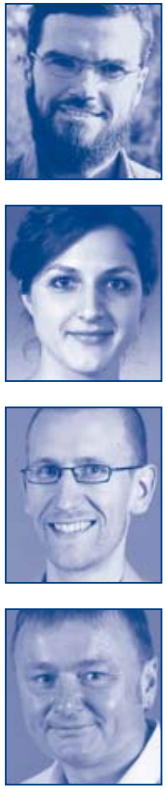